\title{
The PDFLattice2017 workshop: a summary report
}

\author{
Emanuele R. Nocera* \\ Rudolf Peierls Centre for Theoretical Physics, University of Oxford, \\ 1 Keble Road, Oxford OX1 3NP, United Kingdom \\ E-mail: emanuele.nocera@physics.ox.ac.uk
}

\section{Huey-Wen Lin}

Department of Computational Mathematics, Science and Engineering, and

Department of Physics and Astronomy, Michigan State University, East Lansing, MI 48824, USA

\section{Fred Olness}

Department of Physics, Southern Methodist University, Box 0175 Dallas, TX 75275, USA

\section{Kostas Orginos}

Department of Physics, College of William and Mary, Williamsburg, VA 23187-8795, and

Jefferson Laboratory, 12000 Jefferson Avenue, Newport News, VA 23606, USA

\section{Juan Rojo}

Department of Physics and Astronomy, VU University, NL-1081 HV Amsterdam, and Nikhef Theory Group, Science Park 105, 1098 XG Amsterdam, The Netherlands

The workshop on Parton Distributions and Lattice Calculations in the LHC era (PDFLattice2017) was hosted at Balliol College, Oxford (UK), from $22^{\text {nd }}$ to $24^{\text {th }}$ March 2017. The workshop brought together the lattice-QCD and the global-fit physicists who devote their efforts to determine the parton distribution functions (PDFs) of the proton. The goals were to make the two communities more familiar between each other, review developments from both sides, and set precision targets for lattice calculations so that they can contribute, together with the forthcoming experimental input, to the next generation of PDF determinations. This contribution summarises the relevant outcome of the workshop, in anticipation of a thorough white paper.

XXV International Workshop on Deep-Inelastic Scattering and Related Subjects 3-7 April 2017

University of Birmingham, UK

\footnotetext{
* Speaker.
} 
In Quantum Chromodynamics (QCD), Parton Distribution Functions (PDFs) are universal objects that encode the long-distance dynamics of quarks and gluons interacting in a hard-scattering process. Following factorisation [1], they are convoluted with partonic cross sections, that encode instead the short-distance dynamics of the interaction, in order to obtain predictions for the experimental observables. While partonic cross sections can be computed in QCD as a perturbative expansion in the strong coupling $\alpha_{s}$, PDFs cannot, although their dependence on the factorisation scale $\mu$ results in the perturbatively computable DGLAP evolution equations $[2,3,4,5]$.

The accurate knowledge of PDFs is at the base of the understanding of the nucleon structure, including how its momentum and spin are carried by quarks and gluons. Parton distributions are also fundamental tools in high-energy, nuclear and astroparticle phenomenology. Their determination has thus received considerable theoretical and experimental attention over the years $[6,7]$.

There are currently two main methods to determine PDFs. The first method consists in a global QCD analysis of measurements of various hard-scattering observables. Parton distributions are parametrised at an initial scale, evolved up to the scale of the data, and used to build up the theoretical predictions for the relevant observables. In the corresponding factorisation formulæ, the factorisation scale, $\mu$, is usually set equal to the characteristic scale of the process, $Q$. The best-fit parameters are then determined by minimising a suitable figure of merit, such as the $\chi^{2}$.

Several theoretical and methodological details must be handled in a global QCD analysis. On the theoretical side, general physical constraints must be enforced, specifically PDFs must lead to positive cross sections, and fulfill sum rules; heavy quarks must be treated through an appropriate flavour scheme, possibly extended to allow for intrinsic components [8, 9, 10]; and the highest-order QCD corrections should be included in the evolution and in the computation of the partonic cross sections. These are currently available at next-to-next-to-leading order (NNLO) in $\alpha_{s}$ for an increasing number of processes where the polarisation of the initial state is not measured, otherwise they are usually known only up to next-to-leading order (NLO). As the precision of the measurements will increase, PDFs including Quantum Electrodynamics (QED) corrections [11], resummation effects [12], and higher-twist contributions [13,14] might be considered.

On the methodological side, particular attention is devoted to the determination of the PDF uncertainty, which is usually quantified with either the Hessian [15] or the Monte Carlo [16] method. Both these methods allow one to account for various contributions to the PDF uncertainty: the measurement uncertainty propagated from the data, uncertainties associated with incompatibility of the fitted experiments, and procedural uncertainties such as those related to the choice of the PDF parametrisation. Theoretical uncertainties, such as the parametric uncertainty due to the uncertainties on the values of the physical parameters used in the fit (e.g. the reference value of $\alpha_{s}$ ), and the missing higher order uncertainty (given that fits are performed with fixed-order perturbation theory), are instead more elusive. While the size of the former can be estimated by varying the input parameters, the size of the latter is currently unknown, although it is supposed to be subdominant.

Several collaborations provide regular updates of PDF determinations from a global QCD analysis, both in the helicity-averaged (unpolarised, henceforth) $[13,17,18,19,20]$ and helicitydependent (polarised, henceforth) [14, 22, 23] cases. Differences in both the PDF central value and uncertainty from different sets are observed, mostly depending on the data set, and on the theoretical and methodological details of each QCD analysis. In the unpolarised case, most of these differences [24] are now understood, up to a point that some sets [17, 18, 21] were statistically 
combined into a single PDF set [25], with PDFs accurate to a few percent. In the polarised case, instead, a more limited and less precise data set, along with a lower degree in the theoretical and methodological sophistication of the available QCD analyses, has led to less accurate PDFs. This has also prevented from benchmarking various polarised PDF sets quantitatively so far.

The second method is provided by lattice QCD, i.e., QCD formulated on a finite-volume Euclidean spacetime discretised by means of the introduction of an ultraviolet cutoff. Lattice QCD is generally studied by numerical computations of QCD correlation functions in the path-integral formalism, using methods adapted from statistical mechanics. In oder to make contact with the data, numerical results have to be extrapolated to the continuum and infinite-volume limits.

Lattice-QCD calculations primarily determine the matrix elements of local twist-two operators that can be related to the Mellin moments of PDFs. In principle, given a sufficient number of Mellin moments, PDFs can be reconstructed from the inverse Mellin transform. In practice, calculations are limited to the lowest three moments [26, 27, 28, 29, 30, 31, 32, 33, 34], because power-divergent mixing occurs between twist-two operators. Three moments are not enough to reconstruct the momentum fraction dependence of the PDFs without a significant model bias [35]. Novel strategies have been developed to compute higher moments [36, 37], although they are still in their infancy.

Alternative methods have been recently proposed to determine the PDF momentum fraction dependence directly from lattice QCD, among which the inversion method and quasi-PDFs.

The inversion method allows one to relate the unpolarised and polarised structure function $F_{1}$ and $g_{1}$ to the appropriate Compton amplitude through an integral equation, which can be solved numerically. The Compton amplitude can be obtained by a simple extension [38] of existing implementations of the Feynamn-Hellman technique to lattice QCD [39, 40, 41]. Contributions from up, down and strange quarks, connected and disconnected, can be distinguished by appropriate insertions of the electromagnetic current. The same method can be extended to PDFs, provided that $Q$ is sufficiently large that power corrections in the Compton amplitude can be neglected.

Quasi-PDFs [42] are defined as appropriate momentum-dependent nonlocal static matrix elements for nucleon states at finite momentum, with an ultraviolet cut-off scale such as the inverse of the lattice spacing. Quasi-PDFs must be related to the corresponding light-front PDFs, for which the nucleon momentum is taken to infinity. This is usually achieved in the Large-Momentum Effective field Theory (LaMET) [42] by means of a matching kernel. There are approaches alternative to LaMET [43], which view quasi-PDFs as a lattice cross section from which the light-front PDF can be factorised, with related constructions proposed in [44] and explored in [45]. Preliminary results from lattice calculations of quasi-PDFs have been ecouraging, although they are still rather qualitative [46, 47, 48, 49].

In order to make meaningful contact with the data, lattice QCD calculations must demonstrate control over a wide range of systematic uncertainties introduced by the discretisation of QCD on the lattice. These include discretisation effects that vanish in the continuum limit, extrapolation from unphysically pion masses, finite volume effects, excited state contamination, and renormalisation of composite operators. The continuum limit also requires an accurate determination of the lattice spacing, which however introduces a negligible uncertainty. All these sources of systematics are critically reviewed in [50]. In addition, quasi-PDFs are subject to uncertainties associated with the finite nucleon momentum of the lattice calculation, and with their specific renormalisation.

The workshop on Parton Distributions and Lattice Calculations in the LHC era (PDFLat- 


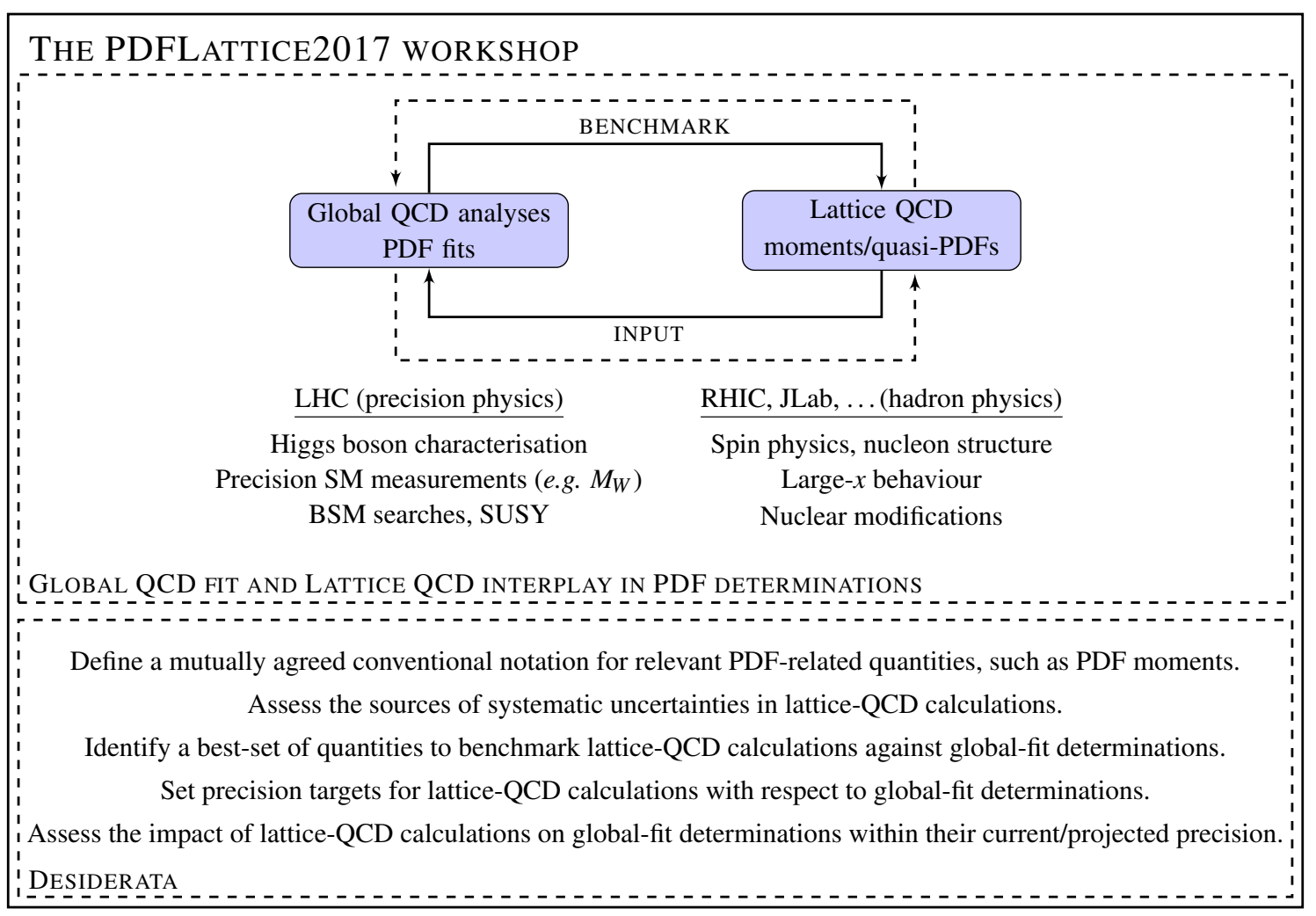

Figure 1: A graphical summary of the PDFLattice2017 workshop outcome.

tice2017) [51] was organised to bring together physicists who actively work to determine PDFs either from global fits or from lattice QCD. It was hosted at Balliol College, Oxford (UK), from $22^{\text {nd }}$ to $24^{\text {th }}$ March 2017. The goals were to make the global-fit and lattice-QCD communities more familiar between each other, review recent developments from both sides, and discuss how lattice-QCD calculations can be used to improve global fits, and, conversely, how global fits can be used to benchmark lattice-QCD calculations. The workshop was specifically focused on precision physics, and included aspects of both the high-energy physics program at the Large Hadron Collider (LHC), and the hadron physics program at the Relativistic Heavy Ion Collider (RHIC), at Jefferson Lab (JLab), and at other facilities. Therefore, the discussion was consciously limited to collinear unpolarised and polarised PDFs. Future editions of the workshop could be extended to transversity, Transverse-Momentum-Dependent PDFs (TMDs), and Generalised PDFs (GPDs).

A graphical summary of the workshop outcome is displayed in Fig. 1. It includes the following list of desiderata, which was compiled in order to strengthen the cross-talk between the two PDF communities in the future. First, define a common language, including a mutually agreed conventional notation for relevant PDF-related quantities, such as PDF moments. Second, assess the sources of systematic uncertainties in lattice-QCD calculations, e.g. along the lines of [50]. Third, indentify a set of quantities to benchmark lattice-QCD results against global-fit determinations, and provide a prescription to select and/or combine the most reliable and up-to-date results from both sides. Fourth, set precision targets for lattice-QCD calculations so that they can contribute, together with the forthcoming experimental input, to the next generation of PDF determinations. 
Fifth and last, assess the impact of lattice-QCD calculations on global-fit determinations assuming their current and/or projected precision.

All these points will be addressed thoroughly in a forthcoming white paper [52]. In particular, this will include a fulll review of recent developments in lattice-QCD and global-fit PDF determinations, a complete set of up-to-date benchmark numbers for the relevant moments from both sides, and an assessment of the impact of lattice-QCD calculations on global-fit determinations assuming their current and/or projected precision. The white paper will represent the joint effort of the global-fit and lattice-QCD PDF communities to make the most of the lively activities spurred by the workshop. It will hopefully become a reference and a further motivation to encourage fruitful interactions between the two communities.

\section{Acknowledgements}

I would like to thank all the participants to the PDFLattice2017 workshop for making it very fruitful. I am also grateful to Jacqueline Gills and Michelle Bosher for their help in the organisation of the workshop. The workshop was partly supported by the European Research Council via the Starting Grant PDF4BSM - Parton Distributions in the Higgs Boson Era. I acknowledge financial support from the UK STFC Rutherford Grant ST/M003787/1.

\section{References}

[1] J. C. Collins, D. E. Soper and G. F. Sterman, Adv. Ser. Direct. High Energy Phys. 5 (1989) 1.

[2] V. N. Gribov and L. N. Lipatov, Sov. J. Nucl. Phys. 15 (1972) 438 [Yad. Fiz. 15 (1972) 781].

[3] L. N. Lipatov, Sov. J. Nucl. Phys. 20 (1975) 94 [Yad. Fiz. 20 (1974) 181].

[4] G. Altarelli and G. Parisi, Nucl. Phys. B 126 (1977) 298.

[5] Y. L. Dokshitzer, Sov. Phys. JETP 46 (1977) 641 [Zh. Eksp. Teor. Fiz. 73 (1977) 1216].

[6] S. Forte and G. Watt, Ann. Rev. Nucl. Part. Sci. 63 (2013) 291.

[7] P. Jimenez-Delgado, W. Melnitchouk and J. F. Owens, J. Phys. G 40 (2013) 093102.

[8] R. D. Ball, M. Bonvini and L. Rottoli, JHEP 1511 (2015) 122.

[9] R. D. Ball et al., Phys. Lett. B 754 (2016) 49.

[10] R. D. Ball et al. [NNPDF Collaboration], Eur. Phys. J. C 76 (2016) 647.

[11] R. D. Ball et al. [NNPDF Collaboration], Nucl. Phys. B 877 (2013) 290.

[12] M. Bonvini et al., JHEP 1509 (2015) 191; L. Rottoli and M. Bonvini, arXiv:1707.01535 [hep-ph].

[13] A. Accardi et al. Phys. Rev. D 93 (2016) 114017.

[14] N. Sato et al. [JAM Collaboration], Phys. Rev. D 93 (2016) 074005.

[15] J. Pumplin et al. Phys. Rev. D 65 (2001) 014013.

[16] L. Del Debbio et al. [NNPDF Collaboration], JHEP 0503 (2005) 080.

[17] L. A. Harland-Lang et al., Eur. Phys. J. C 75 (2015) 204.

[18] S. Dulat et al., Phys. Rev. D 93 (2016) 033006. 
[19] R. D. Ball et al. [NNPDF Collaboration], arXiv:1706.00428 [hep-ph].

[20] S. Alekhin, J. Blümlein, S. Moch and R. Placakyte, Phys. Rev. D 96 (2017) 014011.

[21] R. D. Ball et al. [NNPDF Collaboration], JHEP 1504 (2015) 040.

[22] D. de Florian, R. Sassot, M. Stratmann and W. Vogelsang, Phys. Rev. Lett. 113 (2014) 012001.

[23] E. R. Nocera et al. [NNPDF Collaboration], Nucl. Phys. B 887 (2014) 276.

[24] S. Alekhin et al., arXiv:1101.0536 [hep-ph]; R. D. Ball et al., JHEP 1304 (2013) 125.

[25] J. Butterworth et al., J. Phys. G 43 (2016) 023001.

[26] C. Alexandrou et al., arXiv:1706.02973 [hep-lat].

[27] J. R. Green et al., Phys. Lett. B 734 (2014) 290; G. S. Bali et al., Phys. Rev. D 90 (2014) 074510.

[28] M. Engelhardt, Phys. Rev. D 86 (2012) 114510.

[29] M. Gong et al. [ $\chi$ QCD Collaboration], Phys. Rev. D 95 (2017) 114509.

[30] Y. Aoki et. al., Phys. Rev. D 82 (2010) 014501.

[31] J. D. Bratt et al. [LHPC Collaboration], Phys. Rev. D 82 (2010) 094502.

[32] A. Abdel-Rehim et al., Phys. Rev. D 92 (2015) 114513; Erratum: [Phys. Rev. D 93 (2016) 039904].

[33] T. Bhattacharya et al., Phys. Rev. D 94 (2016) 054508.

[34] S. Capitani et al., arXiv:1705.06186 [hep-lat]; G. S. Bali et al., Phys. Rev. D 91 (2015) 054501.

[35] W. Detmold, W. Melnitchouk and A. W. Thomas, Mod. Phys. Lett. A 18 (2003) 2681.

[36] Z. Davoudi and M. J. Savage, Phys. Rev. D 86 (2012) 054505.

[37] C. Monahan and K. Orginos, Phys. Rev. D 91 (2015) 074513.

[38] A. J. Chambers et al., Phys. Rev. Lett. 118 (2017) 242001.

[39] R. Horsley et al. [QCDSF and UKQCD Collaborations], Phys. Lett. B 714 (2012) 312.

[40] A. J. Chambers et al. [CSSM and QCDSF/UKQCD Collaborations], Phys. Rev. D 90 (2014) 014510.

[41] A. J. Chambers et al., Phys. Rev. D 92 (2015) 114517.

[42] X. Ji, Phys. Rev. Lett. 110 (2013) 262002; Sci. China Phys. Mech. Astron. 57 (2014) 1407.

[43] Y. Q. Ma and J. W. Qiu, arXiv:1404.6860 [hep-ph]; Int. J. Mod. Phys. Conf. Ser. 37 (2015) 1560041.

[44] A. Radyushkin, Phys. Lett. B 767 (2017) 314; arXiv:1705.01488 [hep-ph].

[45] K. Orginos, A. Radyushkin, J. Karpie and S. Zafeiropoulos, arXiv:1706.05373 [hep-ph].

[46] H. W. Lin et al., Phys. Rev. D 91 (2015) 054510.

[47] C. Alexandrou et al., Phys. Rev. D 92 (2015) 014502; Phys. Rev. D 96 (2017) 014513.

[48] J. W. Chen et al., Nucl. Phys. B 911 (2016) 246; arXiv:1706.01295 [hep-lat].

[49] H. W. Lin et al., arXiv:1708.05301 [hep-lat].

[50] S. Aoki et al., Eur. Phys. J. C 77 (2017) 112.

[51] See the workshop web page http://www.physics.ox.ac.uk/confs/PDFlattice2017/index.asp.

[52] H.-W. Lin, E. R. Nocera, F. Olness, K. Orginos, J. Rojo (editors) et al., in preparation. 